\title{
Strongly $\mathcal{P}$-projective Modules and $\mathcal{P}$-projective Complexes
}

\author{
Liang Yan \\ College of Mathematics and Physics Science, Hunan University of Arts and Science, Changde, P. R. China
}

\section{Email address:}

lanyuyanliang@163.com

\section{To cite this article:}

Liang Yan. Strongly $\mathcal{P}$-projective modules and $\mathcal{P}$-projective Complexes. International Journal of Theoretical and Applied Mathematics. Vol. 5, No. 6, 2019, pp. 118-124. doi: 10.11648/j.ijtam.20190506.16

Received: October 30, 2019; Accepted: November 26, 2019; Published: December 19, 2019

\begin{abstract}
In this paper we first study the properties of Strongly $\mathcal{P}$-projective modules, and obtain some equivalent conclusions about Strongly $\mathcal{P}$-projective modules, it is proved that a finitely generated right $R$-module $N$ is strongly $\mathcal{P}$-projective if and only if $\operatorname{Ext}^{i}(N, R)=0$ for all $i \geq 1$ over left noetherian and right perfect ring, a $\mathcal{P}$-projective right $R$-module $N$ is strongly $\mathcal{P}$ projective if and only if the first syzygy of $N$ is strongly $\mathcal{P}$-projective. Then we extend the notion of $\mathcal{P}$-projective modules to that of $\mathcal{P}$-projective complexes. We study the relationships between $\mathcal{P}$-projective complexes and $\mathcal{P}$-projective modules, it is proved that a complex $C$ is $\mathcal{P}$-projective if and only if every $C^{i}$ is $\mathcal{P}$-projective for every integer $i$ if and only if $E x t^{1}(C, P)=0$ for every projective complex $P$ if and only if for every exact sequence $0 \rightarrow A \rightarrow P \rightarrow C \rightarrow 0$ with $P$ projective, $A \rightarrow P$ is a projective preenvelope of $A$. Some characterizations of $\mathcal{P}$-projective complexes also obtained.
\end{abstract}

Keywords: Strongly $\mathcal{P}$-projective Module, $\mathcal{P}$-projective Module, $\mathcal{P}$-projective Complex

\section{Introduction}

Throughout this paper, $R$ is an associative ring with identity. By module we mean right $R$-module. $\mathcal{C}$ will be an abelian category of complexes of right $R$-modules. This category has enough projectives and injectives. This can be seen from the fact that any complex of the form

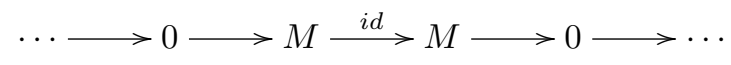

with $M$ projective (injective) is projective (injective). For objects $C$ and $D$ of $\mathcal{C}, \operatorname{Hom}(C, D)$ is the abelian group of morphisms from $C$ to $D$ in $\mathcal{C}$ and $\operatorname{Ext}^{i}(C, D)$ for $i \geq 0$ will denote the groups we get from the right derived functor of Hom.

In this paper, a complex

$$
\cdots \longrightarrow C^{-1} \stackrel{\delta^{-1}}{\longrightarrow} C^{0} \stackrel{\delta^{0}}{\longrightarrow} C^{1} \stackrel{\delta^{1}}{\longrightarrow} \cdots
$$

will be denoted $C$. We will use subscripts to distinguish complexes. So if $\left\{C^{i}\right\}_{i \in I}$ is a family of complexes, $C_{i}$ will be

$$
\cdots \longrightarrow C_{i}^{-1} \stackrel{\delta^{-1}}{\longrightarrow} C_{i}^{0} \stackrel{\delta^{0}}{\longrightarrow} C_{i}^{1} \stackrel{\delta^{1}}{\longrightarrow} \cdots
$$

Given a module $M$, we will denote by $\bar{M}$ the complex

$$
\cdots \longrightarrow 0 \longrightarrow M \stackrel{i d}{\longrightarrow} M \longrightarrow 0 \longrightarrow \cdots
$$

with the $M$ in the -1 and 0 th position. Also we mean by $\underline{M}$ the complex with $M$ in the 0th place and 0 in the other places. Given a complex $C$ and an integer $m, C[m]$ denotes the complex such that $C[m]^{n}=C^{m+n}$ and whose boundary operators are $(-1)^{m} \delta^{m+n}$. If $C$ is a complex we let $Z(C)$ and $B(C)$ be the subcomplex of cycles and boundaries of $C$ and we let $H(C)=Z(C) / B(C)$.

If $f: C \rightarrow D$ is a map of complexes, we can form $M(f)$, the mapping cone of $f, M(f)$ is a complex such that $M(f)^{n}=$ $D^{n} \oplus C^{n+1}$ is mapped to $\left(\delta^{n}(x)+f(x),-\delta^{n+1}(y)\right)$. It is easy to check that there is an exact sequence of complexes $0 \rightarrow D \rightarrow M(f) \rightarrow C[1] \rightarrow 0$.

$\underline{\operatorname{Ext}^{i}}(C, D)$ is the complex 


$$
\cdots \longrightarrow \operatorname{Ext}^{i}(C, D[n-1]) \longrightarrow \operatorname{Ext}^{i}(C, D[n]) \longrightarrow \operatorname{Ext}^{i}(C, D[n+1]) \longrightarrow \cdots,
$$

With boundary operator induced by the boundary operator of $D[9]$. let $\mathcal{F}$ be a class of objects of abelian category $\mathcal{C}$. We donote by $\perp^{\mathcal{F}}$ the left orthogonal class of $\mathcal{F}$, where ${ }^{\perp} \mathcal{F}=\left\{G: \operatorname{Ext}^{1}(G, F)=0\right.$, for all $\left.F \in \mathcal{F}\right\}$, and denote by $\mathcal{F}^{\perp}$ the right orthogonal class of $\mathcal{F}$, where $\mathcal{F}^{\perp}=\{G$ : $\operatorname{Ext}^{1}(F, G)=0$, for all $\left.F \in \mathcal{F}\right\}$.

Let $C$ be a object of $\mathcal{C}$, Recalled that a morphism $f: C \rightarrow F$ with $F \in \mathcal{F}$ is called an $\mathcal{F}$-preenvelope of $C$ if for any morphism $g: C \rightarrow F^{\prime}$ with $F^{\prime} \in \mathcal{F}$, there is a morphism $\theta: F \rightarrow F^{\prime}$ such that $\theta f=g$, moreover, when $F^{\prime}=F$ and $g=f$ the only such $\theta$ are automorphisms of $F$, then $f: C \rightarrow F$ is called an $\mathcal{F}$-envelope of $C$, A monomorphism $f: C \rightarrow F$ is said to be a special $\mathcal{F}$-preenvelope of $C$ if coker $(f) \in^{\perp} \mathcal{F}$. Dually, we have the concepts of (special) $\mathcal{F}$-precover and $\mathcal{F}$-cover. A pair $(\mathcal{F}, \mathcal{G})$ is called a cotorsion theory, if $\mathcal{F}^{\perp}=\mathcal{G}$ and ${ }^{\perp} \mathcal{G}=\mathcal{F}$. A cotorsion theory $(\mathcal{F}, \mathcal{G})$ is called perfect if every object of $\mathcal{C}$ has a $\mathcal{F}$-cover and a $\mathcal{G}$ envelope. A cotorsion theory $(\mathcal{F}, \mathcal{G})$ is called hereditary if $\mathcal{G}$ is resolving.

Recalled that a complex $P$ is projective if and only if it is exact and every $\operatorname{Ker} \delta^{i}$ is a projective module for all $i \in \mathcal{Z}$, a complex $E$ is called a \#-projective complex if all terms $E^{i}$ are projective.

A module $M$ is said to be $\mathcal{P}$-projective if it is a coker of a projective preenvelope [3]. These modules were discovered when studying projective (pre)envelopes. We note that the notion of $P$-projectivity is dual to that of copure injectiveness defined by Enochs and Jenda in [4].

A module $M$ is said to be copure flat if it is flat with respect to the exact sequence $0 \rightarrow A \rightarrow B \rightarrow B / A \rightarrow 0$ with $B / A$ injective[5]. It is easy to see that $M$ is copure flat if and only if $\operatorname{Tor}_{1}(M, E)=0$ for all injective right $R$-module. We will say that $M$ is strongly copure flat if $\operatorname{Tor}_{i}(M, E)=0$ for all injective right $R$-module and all $i \geq 1$.

In section 2 , we introduce the strongly $\mathcal{P}$-projective $R$ module, some characterizations of strongly $\mathcal{P}$-projective $R$ module are given. We also study $\mathcal{P}$-projective dimensions which is based on a similar idea due to [5].

The main purpose of section 3 is to extend the notions of $\mathcal{P}$-projective modules to that of copure injective $\mathcal{P}$-projective complexes . A complex $C$ is said to be $\mathcal{P}$-projective if given any morphism $f: A \rightarrow B \rightarrow 0$ with ker $f$ projective and any morphism $g: C \rightarrow B$, there exists a homomorphism $h: C \rightarrow A$ such that the following diagram commutative

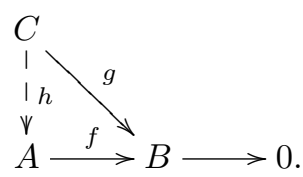

We first obtain a number of characterizations of $\mathcal{P}$ projectivity of complexes. Then It is natural to consider the relationships of $\mathcal{P}$-projectivity of a complex $C$ and $\mathcal{P}$ projectivity of all R-modules $C^{i}$ for $i \in \mathbb{Z}$.
We prove that a complex $C$ is $\mathcal{P}$-projective if and only if every $C^{i}$ is $\mathcal{P}$-projective for $i \in \mathbb{Z}$. Some characterizations of $\mathcal{P}$-projective complexes also obtained.

\section{Strongly $\mathcal{P}$-projective Modules}

In this section we introduce the definitions of strongly $\mathcal{P}$-projective modules, and give some characterizations of strongly $\mathcal{P}$-projective modules.

A right $R$-module $N$ is said to be $\mathcal{P}$-projective if it is a coker of projective (pre)envelope [3]. A right $R$-module $N$ is $\mathcal{P}$ projective if and only if $\operatorname{Ext}^{1}(N, P)=0$ for any projective right $R$-module $P$. We shall say a right $R$-module $N$ is strongly $\mathcal{P}$-projective if $\operatorname{Ext}^{i}(N, P)=0$ for any projective right $R$-module $P$ and all $i \geq 1$.

Remark 2.1 (1)Projective module $\Rightarrow$ Gorenstein projective module $\Rightarrow$ (strongly) $\mathcal{P}$-projective module.

(2) The class of (strongly) $\mathcal{P}$-projective modules is closed under extensions, direct sums and direct summands.

(3) It is easy to see that $N$ is $\mathcal{P}$-projective if and only if given any homomorphism $f: A \rightarrow B \rightarrow 0$ with ker $f$ projective and any homomorphism $g: N \rightarrow B$, there exists a homomorphism $h: N \rightarrow$ A such that the following diagram commutative

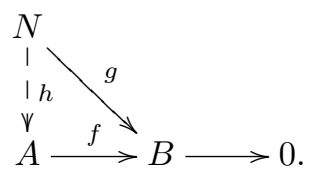

Proposition 2.2 The following are equivalent for a right $R$ module $N$ :

(1) $N$ is projective.

(2) $N$ is $\mathcal{P}$-projective and $p d(N) \leq 1$.

(3) $N$ is strongly $\mathcal{P}$-projective and $p d(N) \leq 1$.

Proof. (1) $\Rightarrow(2)$ is trivial.

$(2) \Rightarrow(1)$ Let $N$ be a $\mathcal{P}$-projective module and $p d(N) \leq 1$. Then there exists an exact sequence $0 \rightarrow K \rightarrow P \rightarrow N \rightarrow 0$. Note that $K$ is projective since $p d(N) \leq 1$. So $\operatorname{Ext}^{1}(N, K)=$ 0 , the above exact sequence splits. Thus $N$ is projective.

$(1) \Rightarrow(3)$ is trivial.

$(3) \Rightarrow(1)$ is similar to $(2) \Rightarrow(1)$.

Proposition 2.3 Let $R$ be a left coherent and right perfect ring. Then the following results are true:

(1) Every (strongly) $\mathcal{P}$-projective right $R$-module is (strongly) copure flat.

(2)Every finitely presented (strongly) copure flat right $R$ module is (strongly) $\mathcal{P}$-projective.

Proof. (1) Let $E$ be an injective left $R$-module, then $E^{+}$is flat since $R$ is left coherent ring, and so $E^{+}$is projective since $R$ is right perfect ring. Thus (1) follows from the standard isomorphism $\operatorname{Ext}^{1}\left(N, M^{+}\right) \cong \operatorname{Tor}_{1}(N, M)^{+}$, where $N$ is any left $R$-module and $M$ is any right $R$-module. 
(2) Let $N$ be a finitely presented (strongly) copure flat right $R$-module. Then there exists a standard isomorphism $\operatorname{Ext}^{i}(N, P)^{+} \cong \operatorname{Tor}_{i}\left(M, P^{+}\right)$. Note that any projective right $R$-module $P$ is flat since $R$ is a right perfect ring, and so $P^{+}$ is injective. Thus the result holds.

Proposition 2.4 Let $R$ be a left noetherian and right perfect ring. Then a finitely generated right $R$-module $N$ is strongly $\mathcal{P}$-projective if and only if $\operatorname{Ext}^{i}(N, R)=0$ for all $i \geq 1$.

Proof. If $N$ is strongly $\mathcal{P}$-projective, then $\operatorname{Ext}^{i}(N, R)=0$ for all $i \geq 1$ since $R$ is projective as a right $R$-module. Conversely, Let $P$ be a projective right $R$-module, $P$ is flat since $R$ is a right perfect ring. By Theorem 4.34 of [11], $P$ is a direct limit of finitely generated free modules, $\operatorname{Ext}^{i}(N, P)=$ 0 by Lemma 3.1.16 of [6] since $R$ is left noetherian and $N$ is finitely generated.

Corollary 2.5 Let $R$ be a left noetherian and right perfect ring. Then a finitely generated right $R$-module $N$ is $\mathcal{P}$ projective if and only if $\operatorname{Ext}^{1}(N, R)=0$.

Let $\mathcal{P}_{\mathcal{P}}$ (resp., $S \mathcal{P}_{\mathcal{P}}$ ) denote the class of $\mathcal{P}$-projective (resp., strongly $\mathcal{P}$-projective ) right $R$-modules. Proposition 2.6 Let $R$ be a left coherent and right perfect ring, then:

(1) $\left(\mathcal{P}_{\mathcal{P}}, \mathcal{P}_{\mathcal{P}}{ }^{\perp}\right)$ is a perfect cotorsion theory.

(2) $\left(\mathcal{S} \mathcal{P}_{\mathcal{P}}, \mathcal{S} \mathcal{P}_{\mathcal{P}}{ }^{\perp}\right)$ is a perfect hereditary cotorsion theory.

Proof. Since every projective right $R$-module $P$ is pure injective over a left coherent and right perfect ring, $\left(\mathcal{P}_{\mathcal{P}}, \mathcal{P}_{\mathcal{P}}{ }^{\perp}\right)$ is a perfect cotorsion theory by Theorem 2.8 of [14], and $\left(S \mathcal{P}_{\mathcal{P}}, \mathcal{S} \mathcal{P}_{\mathcal{P}}{ }^{\perp}\right)$ is a perfect cotorsion theory by Corollary 3.2.12 of [10].

Let $\mathcal{P}$ denote the class of projective right $R$-module. We have the following : Proposition 2.7 The following are equivalent:

(1) $R$ is a $Q F$ ring.

(2) Every module is $\mathcal{P}$-projective.

(3) Every quotient of a $\mathcal{P}$-projective module is $\mathcal{P}$-projective.

(4) $\left(\mathcal{P}_{\mathcal{P}}, \mathcal{P}\right)$ is a cotorsion theory.

Proof. (1) $\Leftrightarrow(2)$ follows from the fact that $R$ is a $Q F$ ring if and only if every projective module is injective (we also can see Remark 2. 3 of [3])

$(2) \Rightarrow(3)$ is trivial.

$(3) \Rightarrow(2)$ We simply note that every module is a quotient of a $\mathcal{P}$-projective module since every projective module is $\mathcal{P}$ projective.

$(1) \Rightarrow(4)$ is clear.

$(4) \Rightarrow(1) R$ is a $Q F$ ring since every injective module is projective by (4).

An exact sequence $\cdots \rightarrow A_{2} \rightarrow A_{1} \rightarrow A_{0} \rightarrow N \rightarrow 0$ where $A_{0} \rightarrow N, A_{1} \rightarrow \operatorname{ker}\left(A_{0} \rightarrow N\right), A_{n+1} \rightarrow \operatorname{ker}\left(A_{n} \rightarrow\right.$ $\left.A_{n-1}\right)$ are strongly $\mathcal{P}$-projective precovers is called a $\mathcal{P}$ projective resolution of $N$. If there exists a $\mathcal{P}$-projective resolution $0 \rightarrow A_{n} \rightarrow \cdots \rightarrow A_{2} \rightarrow A_{1} \rightarrow A_{0} \rightarrow N \rightarrow 0$, we say that $N$ has $\mathcal{P}$-projective dimension $(\mathcal{P} p d) \leq n$. Let $\mathcal{P} p d(R)=\sup \{\mathcal{P} p d(N) \mid N$ is a right $R$-module $\}$, we call $\mathcal{P} p d(R)$ the $\mathcal{P}$-projective dimension of $R$.

Proposition 2.8 Let $R$ be a left coherent and right perfect ring. Then the following are equivalent for a right $R$-module:

(1) $\mathcal{P} p d(N) \leq n$.
(2) $\operatorname{Ext}^{n+i}(N, P)=0$ for all projective right $R$-module $P$ and all $i \geq 1$.

(3) Every nth syzygy of $N$ is strongly $\mathcal{P}$-projective.

Proof. (1) $\Leftrightarrow(2)$ Let $0 \rightarrow K \rightarrow A_{n-1} \rightarrow \cdots \rightarrow A_{2} \rightarrow$ $A_{1} \rightarrow A_{0} \rightarrow N \rightarrow 0$ be an exact sequence with $A_{0}, A_{1}$, $A_{2}, \cdots, A_{n-1}$ strongly $\mathcal{P}$-projective. Then $\operatorname{Ext}^{i}(K, P) \cong$ $\operatorname{Ext}^{n+i}(N, P)$. Thus the result follows.

$(2) \Leftrightarrow(3)$ is similar to $(1) \Leftrightarrow(2)$.

Remark 2.9 We note that the $\mathcal{P}$-projective dimension of a right $R$-module $N$ can be considered as the largest positive integer $n$ such that $\operatorname{Ext}^{n}(N, P) \neq 0$ for some projective module $P$. Taking this as a definition of $\mathcal{P}$-projective dimension, we may drop the left coherent and right perfect conditions in Proposition above.

Corollary 2.10 Let $R$ be a ring. Then a $\mathcal{P}$-projective right $R$ module $N$ is strongly $\mathcal{P}$-projective if and only if the first syzygy of $N$ is strongly $\mathcal{P}$-projective. Corollary 2.11 if $p d(N)<\infty$, then $\mathcal{P} p d(N)=p d(N)$.

Proof. $\operatorname{Ppd}(N) \leq p d(N)$ follows from Proposition 2.8 since projective modules are $\mathcal{P}$-projective. Suppose $p d(N)=n$, then there exists a right $R$-module $A$ such that $\operatorname{Ext}^{n}(N, A) \neq 0$. For right $R$-module $A$, there is an exact sequence $0 \rightarrow K \rightarrow P \rightarrow A \rightarrow 0$ with $P$ projective. Applying $\operatorname{Hom}(N,-)$ to this exct sequence, we get a long exact sequence $\cdots \rightarrow \operatorname{Ext}^{n}(N, P) \rightarrow \operatorname{Ext}^{n}(N, A) \rightarrow$ $\operatorname{Ext}^{n+1}(N, K) \rightarrow \cdots$, then $\operatorname{Ext}^{n+1}(N, K)=0$ since $p d(N)=n$. But $\operatorname{Ext}^{n}(N, A) \neq 0$, so $\operatorname{Ext}^{n}(N, P) \neq 0$. Thus $\mathcal{P} p d(N) \geq n=p d(N)$ by Remark 2.9.

Proposition 2.12 Let $R$ be a left noetherian and right perfect ring, $N$ a finitely generated right $R$-module and $n$ a nonnegative integer. Then $\mathcal{P} p d(N) \leq n$ if and only if $\operatorname{Ext}^{n+i}(N, R)=0$ for all $i \geq 1$.

Proof. Similar to the proof of Proposition 2.4.

The following proposition is Dual to the equivalences (1)(3) of [5].

Proposition 2.13 The following are equivalent for a left and right noetherian ring $R$ :

(1) $R$ is $n$-Gorenstein.

(2) $\mathcal{P} p d(N) \leq n$ for all $R$-modules (left and right) $N$.

(3) Every nth syzygy of $N$ is strongly $\mathcal{P}$-projective.

Corollary 2.14 The following are equivalent for a two-sided noetherian and perfect ring $R$ :

(1) $R$ is 1-Gorenstein.

(2) $\mathcal{P} p d(N) \leq 1$ for all $R$-module (left and right) $N$.

(3) Every $\mathcal{P}$-projective $R$-module (left and right) is strongly $\mathcal{P}$-projective.

(4) Every submodule of a strongly $\mathcal{P}$-projective $R$-module (left and right) is strongly $\mathcal{P}$-projective.

Proof. (1) $\Leftrightarrow(2)$ follows from the Proposition above.

$(2) \Rightarrow(3)$ Let $N$ be a $\mathcal{P}$-projective $R$-module. Then the first syzygy of $N$ is strongly $\mathcal{P}$-projective by Proposition 2.8 , and so $N$ is strongly $\mathcal{P}$-projective by Corollary 2.10 .

$(3) \Rightarrow(2)$ Let $N$ be an $R$-module. Then there exists an exact sequence $0 \rightarrow K \rightarrow P \rightarrow N \rightarrow 0$ with $P$ projective. Let $K \rightarrow P^{\prime}$ be a projective preenvelope which is monic since $K$ is a submodule of projective. Note that $L=\operatorname{coker}(K \rightarrow$ 
$\left.P^{\prime}\right)$ is $\mathcal{P}$-projective by the definition of $\mathcal{P}$-projective modules, and so $L$ is strongly $\mathcal{P}$-projective by (3). Hence $K$ is strongly $\mathcal{P}$-projective, as desired.

$(2) \Rightarrow(4)$ Let $M$ be a submodule of a strongly $\mathcal{P}$-projective $R$-module $N$. Applying the functor $\operatorname{Hom}(-, P)$ to the exact sequence $0 \rightarrow M \rightarrow N \rightarrow N / M \rightarrow 0$, we obtain an exact sequence $0=\operatorname{Ext}^{i}(N, P) \rightarrow \operatorname{Ext}^{i}(M, P) \rightarrow E x t^{1+i}$ for all $i \geq 1$. But the last term is zero by (2), so $\operatorname{Ext}^{i}(M, P)=0$ for all $i \geq 1$, as desired.

$(4) \Rightarrow(2)$ is obvious.

\section{3. $\mathcal{P}$-projective Complexes}

Definition 3.1 A complex $C$ is said to be $\mathcal{P}$-projective if given any morphism $f: A \rightarrow B \rightarrow 0$ with ker $f$ projective and any morphism $g: C \rightarrow B$, there exists a morphism $h: C \rightarrow A$ such that the following diagram commutative

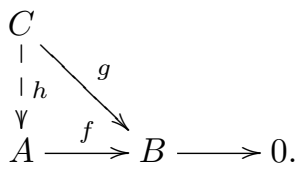
$C$ :

Proposition 3.2 The following are equivalent for a complex

(1) $C$ is $\mathcal{P}$-projective.

(2) $\operatorname{Ext}^{1}(C, P)=0$ for every projective complex.

(3) For every exact sequence $0 \rightarrow A \rightarrow P \rightarrow C \rightarrow 0$ with $P$ projective, $A \rightarrow P$ is a projective preenvelope of $A$.

(4) $C$ is a coker of a projective preenvelope.

(5) For any short exact sequence $0 \rightarrow A \rightarrow B \rightarrow C \rightarrow 0$ and any projective $P$, the sequence $\operatorname{Hom}(B, P) \rightarrow$ $\operatorname{Hom}(A, P) \rightarrow 0$ is exact.

(6) $\operatorname{Hom}(C,-)$ exact any short exact sequence $0 \rightarrow P \rightarrow$ $A \rightarrow B \rightarrow 0$ with $P$ projective.

Proof. (1) $\Rightarrow(2)$ Let $0 \rightarrow P \rightarrow E \rightarrow N \rightarrow 0$ be a short injective resolution of projective complex $P$. Then we have a long exact sequence $0 \rightarrow \operatorname{Hom}(C, P) \rightarrow \operatorname{Hom}(C, E) \rightarrow$ $\operatorname{Hom}(C, N) \rightarrow \operatorname{Ext}^{1}(C, P) \rightarrow \operatorname{Ext}^{1}(C, E)=0$. But $0 \rightarrow$ $\operatorname{Hom}(C, P) \rightarrow \operatorname{Hom}(C, E) \rightarrow \operatorname{Hom}(C, N) \rightarrow 0$ is exact by definition of $\mathcal{P}$-projective modules. So $\operatorname{Ext}^{1}(C, P)=0$.

$(2) \Rightarrow(1)$ is straightforward.

$(2) \Rightarrow(3)$ Let $P^{\prime}$ be projective, then there is an exact sequence $0 \rightarrow \operatorname{Hom}\left(P, P^{\prime}\right) \rightarrow \operatorname{Hom}\left(A, P^{\prime}\right) \rightarrow$ $\operatorname{Ext}\left(C, P^{\prime}\right)=0$ by (2), so $A \rightarrow P$ is a projective preenvelope.

$(3) \Rightarrow(4)$ is obvious.

$(4) \Rightarrow(2)$ Let $C$ be a coker of a projective preenvelope $f: A \rightarrow P$ with $P$ projective. then there is an exact sequence $0 \rightarrow K \rightarrow P \rightarrow C \rightarrow 0$ with $K=i m(f)$. For each projective module $P^{\prime}$ we have a long exact sequence $\operatorname{Hom}\left(P, P^{\prime}\right) \rightarrow$ $\operatorname{Hom}\left(K, P^{\prime}\right) \rightarrow \operatorname{Ext}^{1}\left(C, P^{\prime}\right) \rightarrow \operatorname{Ext}^{1}\left(P, P^{\prime}\right)=0$. But $\operatorname{Hom}\left(P, P^{\prime}\right) \rightarrow \operatorname{Hom}\left(K, P^{\prime}\right) \rightarrow 0$ is exact by (4). Hence $\operatorname{Ext}^{1}\left(C, P^{\prime}\right)=0$.

$(2) \Rightarrow(5)$ is obvious.
$(5) \Rightarrow(2)$ There exists an exact sequence $0 \rightarrow K \rightarrow P \rightarrow$ $C \rightarrow 0$ with $P$ projective. Then for any projective $P^{\prime}$, we have the exact sequence $\operatorname{Hom}\left(P, P^{\prime}\right) \rightarrow \operatorname{Hom}\left(K, P^{\prime}\right) \rightarrow$ $\operatorname{Ext}^{1}\left(C, P^{\prime}\right) \rightarrow \operatorname{Ext}^{1}\left(P, P^{\prime}\right)=0$. But $\operatorname{Hom}\left(P, P^{\prime}\right) \rightarrow$ $\operatorname{Hom}\left(K, P^{\prime}\right) \rightarrow 0$ is exact, so $\operatorname{Ext}^{1}\left(C, P^{\prime}\right)=0$.

$(2) \Rightarrow(6)$ is obvious.

$(6) \Rightarrow(2)$ Let $P$ be a projective. Then there is an exact sequence $0 \rightarrow P \rightarrow E \rightarrow L \rightarrow 0$, and we have a long exact $0 \rightarrow \operatorname{Hom}(C, P) \rightarrow \operatorname{Hom}(C, E) \rightarrow \operatorname{Hom}(C, L) \rightarrow$ $\operatorname{Ext}^{1}(C, P) \rightarrow \operatorname{Ext}^{1}(C, E)=0$. But $0 \rightarrow \operatorname{Hom}(C, P) \rightarrow$ $\operatorname{Hom}(C, E) \rightarrow \operatorname{Hom}(C, L) \rightarrow 0$ is exact by (6), so $\operatorname{Ext}^{1}(C, P)=0 . \quad \square$ Remark 3.3 (1)Projective complex $\Rightarrow$ Gorenstein projective complex $\Rightarrow \mathcal{P}$-projective complex.

(2) The class of $\mathcal{P}$-projective complexes is closed under extensions, direct sums and direct summands.

(3) If $C$ is a $\mathcal{P}$-projective complex, then $C[i]$ is also a $\mathcal{P}$ projective complex for all $i \in \mathbb{Z}$.

It is natural to consider the relationships of $\mathcal{P}$-projectivity of a complex $C$ and $\mathcal{P}$-projectivity of all modules $C^{i}$ for $i \in \mathbb{Z}$. Next we give the following results.

Theorem 3.4 The following are equivalent for a complex $C$ :

(1) $C$ is $\mathcal{P}$-projective.

(2) $\operatorname{Ext}^{1}(C, \bar{P}[n])=0$ for any projective module $P$.

(3) Every $C^{i}$ is $\mathcal{P}$-projective for $i \in \mathbb{Z}$ and Hom $(C, P)$ is exact for each projective complex $P$.

(4) Every $C^{i}$ is $\mathcal{P}$-projective for $i \in \mathbb{Z}$.

Proof. $(1) \Rightarrow(2)$ For any projective module $P, \bar{P}[n]$ is projective, so $\operatorname{Ext}^{1}(C, \bar{P}[n])=0$ by Proposition 3.2.

$(2) \Rightarrow(1)$ Note that any projective complex is the direct product of complexes

$$
\overline{P_{i}}[i]=\cdots \longrightarrow 0 \longrightarrow P_{i} \stackrel{i d}{\longrightarrow} P_{i} \longrightarrow 0 \longrightarrow \cdots
$$

with $P_{i}$ projective. It is easy to check that $\operatorname{Ext}^{1}(C, P)=0$ for any projective complex $P$. So $C$ is $\mathcal{P}$-projective.

$(1) \Rightarrow(3)$ Let $0 \rightarrow P \rightarrow A \rightarrow C^{i} \rightarrow 0$ be any exact sequence of modules with $P$ projective. Then we get the following pullback diagram:

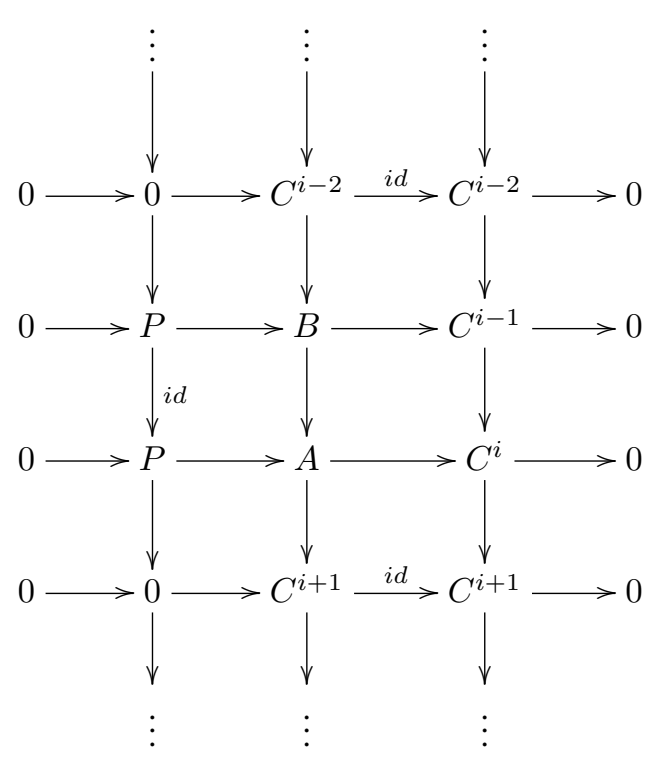


Then $\operatorname{Ext}^{1}(C, \bar{P}[-i])=0$ since $\bar{P}[-i]$ is projective. So the above exact sequence of complexes is split. Thus the exact sequence $0 \rightarrow P \rightarrow A \rightarrow C^{i} \rightarrow 0$ is split, $\operatorname{Ext}^{1}\left(C^{i}, P\right)=0$, so $C^{i}$ is $\mathcal{P}$-projective. For any projective complex $P$, the short exact sequence of complexes $0 \rightarrow P[n] \rightarrow M(f) \rightarrow C[1] \rightarrow$ 0 is split for any $n \in \mathbb{Z}$ and any map $f: C \rightarrow p[n]$ by (1). So $f$ is homotopic to zero by lemma 2.3 .2 of [9]. It is easy to check that $\mathrm{Hom}(C, P)$ is exact.

$(3) \Rightarrow(4)$ is obvious.

$(4) \Rightarrow(2)$ Let

$$
0 \longrightarrow \bar{P}[n] \stackrel{f}{\longrightarrow} A \stackrel{g}{\longrightarrow} C \longrightarrow 0
$$

be any exact sequence of complexes, and we consider the following commutative diagram:

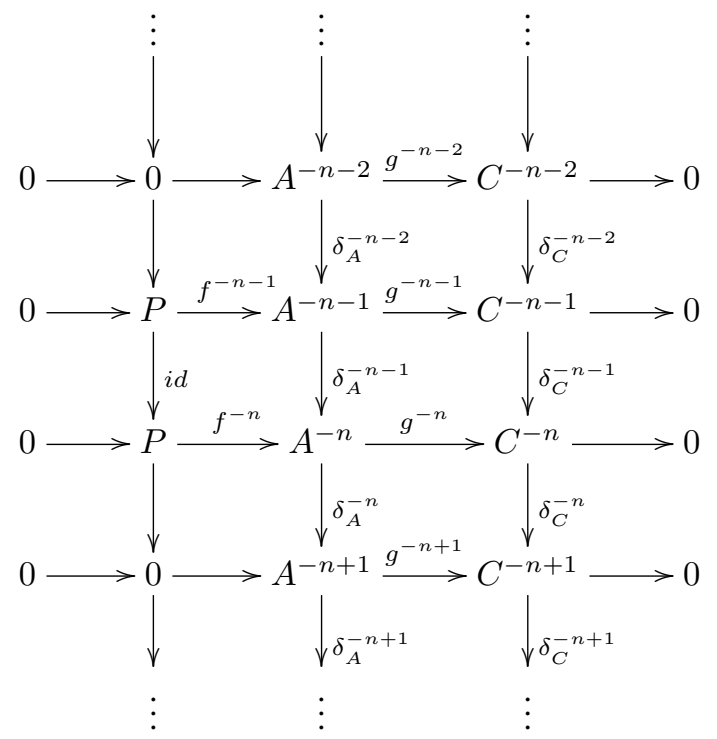

Note that $\operatorname{Ext}^{1}\left(C^{-n}, P\right)=0$ since $C^{-n}$ is $\mathcal{P}$-projective. So $f^{-n}: P \rightarrow A^{-n}$ splits, there exists a morphism $h^{-n}$ : $A^{-n} \rightarrow P$ such that $h^{-n} f^{-n}=i d_{P}$. We define $h^{-n-1}$ : $A^{-n-1} \rightarrow P$ as $h^{-n-1}=h^{-n} \delta_{A}^{-n-1}$ and $h^{i}=0$ for $i \neq-n$, $-n-1$. Then $h=\left\{h^{i}\right\}_{i \in \mathbb{Z}}$ is the morphism from $A$ to $\bar{P}[n]$ such that $h f=i d_{\bar{P}[n]}$. So the sequence

$$
0 \longrightarrow \bar{P}[n] \stackrel{f}{\longrightarrow} A \stackrel{g}{\longrightarrow} C \longrightarrow 0
$$

splits, and so $\operatorname{Ext}^{1}(C, \bar{P}[n])=0$.

Corollary 3.5 The following are equivalent for a module $M$ :

(1) $M$ is $\mathcal{P}$-projective.

(2) $M[n]$ is $\mathcal{P}$-projective for all $n \in \mathbb{Z}$.

(3) $\bar{M}[n]$ is $\mathcal{P}$-projective for all $n \in \mathbb{Z}$.

Corollary 3.6 The following are equivalent for a complex $C$ :

(1) $C$ is $\mathcal{P}$-projective.

(2) Every exact sequence $0 \rightarrow P \rightarrow A \rightarrow C \rightarrow 0$ with $P$ projective splits.

(3) $\operatorname{Ext}(C, P)=0$ for any projective complex $P$.

Recalled that a complex $C$ is said to be \#-projective if every $C^{i}$ is projective for all $i \in \mathbb{Z}$. We have the following Proposition.
Proposition 3.7 The following are equivalent for a complex $C$ :

(1) $C$ is $\mathcal{P}$-projective.

(2) $\operatorname{Ext}^{1}(C, P)=0$ for every $\operatorname{Hom}(Q,-)$ exact bounded \#-projective complex $P$ whenever $Q$ is a $\mathcal{P}$-projective module.

(3) $\operatorname{Ext}^{1}(C, P)=0$ for every $\operatorname{Hom}(Q,-)$ exact bounded above \#-projective complex $P$ whenever $Q$ is a $\mathcal{P}$-projective module.

Proof.(1) $\Rightarrow(3)$ Let $P$ be a $\operatorname{Hom}(Q,-)$ exact bounded above \#-projective complex whenever $Q$ is a $\mathcal{P}$-projective module, without loss of generality, we may assume that $P^{i}=$ 0 for $i>0$. Let

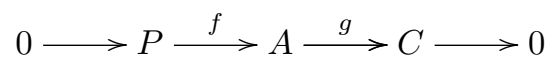

be any exact sequence of complexes. Then we consider the following commutative diagram:

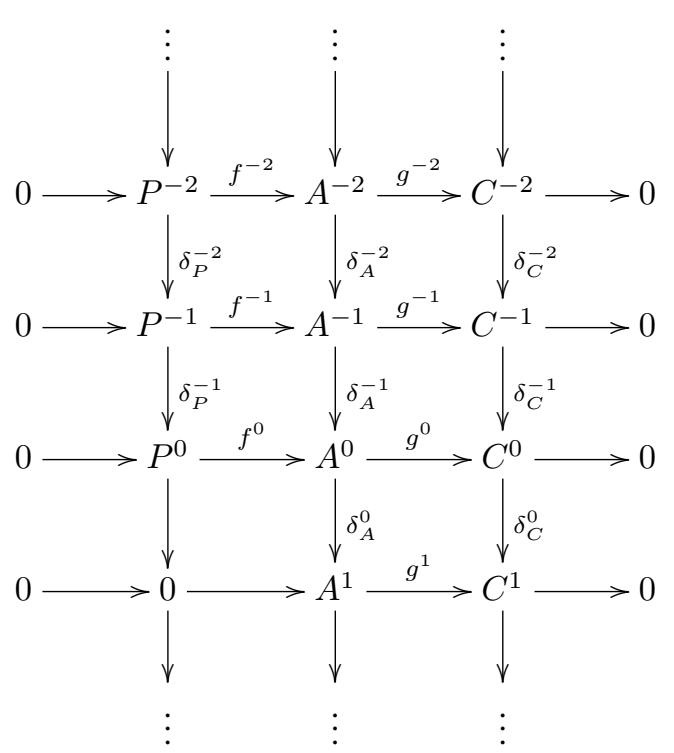

We see that every exact sequence

$$
0 \longrightarrow P^{i} \stackrel{f^{i}}{\longrightarrow} A^{i} \stackrel{g^{i}}{\longrightarrow} C^{i} \longrightarrow 0
$$

is split since $C^{i}$ is $\mathcal{P}$-projective by Theorem 3.4. So there exists $h^{i}: A^{i} \rightarrow P^{i}$ such that $h^{i} f^{i}=i d_{P^{i}}$ for all $i \leq 0$. Now Let $\alpha^{0}=h^{0}$.

Since coker $f^{-1} \cong C^{-1}$, it follows that

$$
\operatorname{Hom}\left(\operatorname{coker} f^{-1}, P^{-1}\right) \rightarrow \operatorname{Hom}\left(\operatorname{coker} f^{-1}, P^{0}\right) \rightarrow 0
$$

is exact. Note that $\left(\delta_{P}^{-1} h^{-1}-\alpha^{0} \delta_{A}^{-1}\right) f^{-1}=\delta_{P}^{-1} h^{-1} f^{-1}-$ $\alpha^{0} \delta_{A}^{-1} f^{-1}=\delta_{P}^{-1}-\alpha^{0} f^{0} \delta_{P}^{-1}=\delta_{P}^{-1}-\delta_{P}^{-1}=0$, so $\overline{\delta_{P}^{-1} h^{-1}-\alpha^{0} \delta_{A}^{-1}} \in \operatorname{Hom}\left(\operatorname{cokerf} f^{-1}, P^{0}\right)$. Thus there exists $\overline{\gamma^{-1}} \in \operatorname{Hom}\left(\operatorname{cokerf} f^{-1}, P^{-1}\right)$ such that $\overline{\delta_{P}^{-1} h^{-1}-\alpha^{0} \delta_{A}^{-1}}=$ $\delta_{P}^{-1} \overline{\gamma^{-1}}$, where $\gamma^{-1} \in \operatorname{Hom}\left(A^{-1}, P^{-1}\right)$ and $\gamma^{-1}\left(P^{-1}\right)=0$. let $\alpha^{-1}=h^{-1}-\gamma^{-1}$, then $\delta_{P}^{-1} \alpha^{-1}=\delta_{P}^{-1} h^{-1}-\delta_{P}^{-1} \gamma^{-1}=$ $\alpha^{0} \delta_{A}^{-1}$, and $\alpha^{-1} f^{-1}=h^{-1} f^{-1}-\gamma^{-1} f^{-1}=h^{-1} f^{-1}=$ $i d_{P^{-1}}$ since $\gamma^{-1} f^{-1}=0$. 
It follows that

$$
\begin{gathered}
\operatorname{Hom}\left(\operatorname{cokerf}^{-2}, P^{-2}\right) \rightarrow \\
\operatorname{Hom}\left(\operatorname{cokerf} f^{-2}, P^{-1}\right) \rightarrow \operatorname{Hom}\left(\operatorname{cokerf} f^{-2}, P^{0}\right)
\end{gathered}
$$

is exact since cokerf $f^{-2} \cong C^{-2}$. Note that $\left(\delta_{P}^{-2} h^{-2}-\right.$ $\left.\alpha^{-1} \delta_{A}^{-2}\right) f^{-2}=\delta_{P}^{-2} h^{-2} f^{-2}-\alpha^{-1} \delta_{A}^{-2} f^{-2}=\delta_{P}^{-2}-$ $\alpha^{-1} f^{-1} \delta_{P}^{-2}=\delta_{P}^{-2}-\delta_{P}^{-2}=0$, then $\overline{\left(\delta_{P}^{-2} h^{-2}-\alpha^{-1} \delta_{A}^{-2}\right)} \in$ $\operatorname{Hom}\left(\operatorname{cokerf} f^{-2}, P^{-1}\right)$, but $\delta_{P}^{-1} \overline{\left(\delta_{P}^{-2} h^{-2}-\alpha^{-1} \delta_{A}^{-2}\right)}=$ 0 , so there exists $\overline{\gamma^{-2}} \in \operatorname{Hom}\left(\operatorname{cokerf} f^{-2}, P^{-2}\right)$ such that $\delta_{P}^{-2} \overline{\gamma^{-2}}=\overline{\left(\delta_{P}^{-2} h^{-2}-\alpha^{-1} \delta_{A}^{-2}\right)}$, where $\gamma^{-2} \in$ $\operatorname{Hom}\left(A^{-2}, P^{-2}\right)$ and $\gamma^{-2}\left(P^{-2}\right)=0$. Now let $\alpha^{-2}=$ $h^{-2}-\gamma^{-2}$, then $\delta_{P}^{-2} \alpha^{-2}=\delta_{P}^{-2} h^{-2}-\delta_{P}^{-2} \gamma^{-2}=\alpha^{-1} \delta_{A}^{-2}$, and $\alpha^{-2} f^{-2}=h^{-2} f^{-2}-\gamma^{-2} f^{-2}=h^{-2} f^{-2}=i d_{P^{-2}}$ since $\gamma^{-2} f^{-2}=0$.

Similarly, we can obtain $\alpha^{-i}: A^{-i} \rightarrow P^{-i}$ such that $\delta_{P}^{-i} \alpha^{-i}=\alpha^{-i+1} \delta_{A}^{-i}$ and $\alpha^{-i} f^{-i}=i d_{P^{-i}}$ for $i \geq 3$. Finally, let $\alpha^{n}=0$ for $n \geq 1$. Then we obtain a morphism $\alpha=\left\{\alpha^{i}\right\}_{i \in \mathbb{Z}}: A \rightarrow P$ such that $\alpha f=i d_{P}$. This implies that the exact sequence

$$
0 \longrightarrow P \stackrel{f}{\longrightarrow} A \stackrel{g}{\longrightarrow} C \longrightarrow 0
$$

splits. Thus $\operatorname{Ext}^{1}(C, P)=0$.

$(3) \Rightarrow(2)$ is obvious.

$(2) \Rightarrow(1)$ Let $P$ be a projective module, $\operatorname{Ext}^{1}(C, \bar{P}[n])=$ 0 by (2), so $C$ is $\mathcal{P}$-projective by Theorem 3.4.

Proposition 3.8 The following are equivalent:

(1) $R$ is a $Q F$ ring.

(2) Every complex is $\mathcal{P}$-projective.

(3) Every quotient of a $\mathcal{P}$-projective complex is $\mathcal{P}$. projective.

Proof. (1) $\Rightarrow(2)$ Let $C$ be a complex, then $C^{i}$ is $\mathcal{P}$ projective by Proposition 2.7. So $C$ is a $\mathcal{P}$-projective complex by Theorem 3.4.

$(2) \Rightarrow(3)$ is obvious.

$(3) \Rightarrow(1)$ Let $M$ be a quotient of a $\mathcal{P}$-projective module $N$. Then $\bar{M}$ is a quotient of a $\mathcal{P}$-projective complex $\bar{N}$, so $\bar{M}$ is $\mathcal{P}$-projective, thus $M$ is $\mathcal{P}$-projective by Theorem 3.4. So $R$ is a $Q F$ ring by Proposition 2.7.

We conclude the paper with the following Remark. Remark 3.9 (1) let $R$ be a left coherent and right perfect ring. Then every right $R$-module has a $\mathcal{P}$-projective cover by Proposition 2.6. So every bounded above complex has a $\mathcal{P}$-projective precover by Proposition 5.3 of [12] since $\mathcal{P}$ projective complexes coincide with \#-P-projective complexes by Theorem 3.4.

(2) If every complex has a projective envelope and we replace "P-projective modules" with "P-projective complexes" in [3], the results still hold by similar proofs.

\section{Conclusion}

The relationship between module and complex is an important research content in homological algebra. We find a new complex called $\mathcal{P}$-projective complex from $\mathcal{P}$-projective module, and reveal the relationship between them. Then we study the properties and homology dimension of $\mathcal{P}$-projective complex in different rings. This kind of work is very meaningful.

\section{Acknowledgements}

The work was supported by the Natural Science Foundation of Hunan Province (grant Nos.2016JJ3095) and the DR. Start -up Funds of Hunan University of Arts and Science(grant Nos. 15BSQD15).

\section{References}

[1] F. W. Anderson, K. R. Fuller, Rings and Categories of Modules, second ed., New York, Spring-verlag, 1992.

[2] L. L. Avramov, H.-B.Foxby, Homological dimensions of unbounded complexes. J. Pure Appl. Algebra 71(1991):129-155.

[3] J. L. Chen, P-Projective modules, Communications in Algebra,24(1996): 3,821-831

[4] E. E. Enochs, O. M. G. Jenda, Copure injective modules, Quaest. Math. 14 (1991) 401-409.

[5] E. E. Enochs, O. M. G. Jenda, Copure injective resolutions, flat resolutions and dimensions, Comment. Math. 34 (1993) 203-211.

[6] E. E. Enochs, O. M. G. Jenda, Relative Homological Algebra, Walter de Gruyter, Berlin, New York, 2000.

[7] E. E. Enochs, L. Oyonarte, Covers, Envelopes and Cotorsion Theories. Nova Science Publishers, Inc. New York, 2002.

[8] E. E. Enochs, S. Estrada, A.Iacob, Gorenstein projective and flat complexes over noetherian rings, Math. Nachr.(2012) 1-18.

[9] J. R. García Rozas, Covers and Envelopes in the Category of Complexes of Modules. Boca RatonLondon-New York-Washington, D. C.: CRC Press, 1999.

[10] R. Göbel, J. Trlifaj, Approximations and Endomorphism Algebras of modules. Berlin-New York: Walter de Gruyter, 2006.

[11] T. Y. Lam, Lectures on modules and rings, New YorkHeidelberg-Berlin: Springer-Verlag, 1999.

[12] L. Li, N. Q. Ding, G.Yang, covers and Envelopes by \#- $\mathcal{F}$ Complexes. Communications in Algebra 39(2011) 32533277. 
[13] L. X. Mao, N. Q. Ding, Relative copure injective and copure flat modules. Journal of Pure and Applied Algebra 208 (2007) 635-646.
[14] J. Trlifaj, Cover, Envelope, and Cotorsion Theories; Lecture notes for the workshop, Homological Methods in Module Theory, Cortona, September 10-16, 2000. 